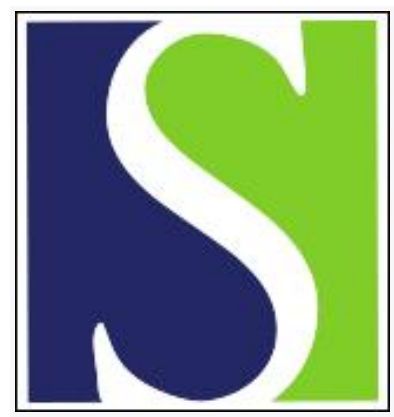

Scand J Work Environ Health 2017;43(5):436-446

https://doi.org/10.5271/sjweh.3655

Published online: 26 Jun 2017, Issue date: 01 Sep 2017

Return to work after work-related stress: a randomized controlled trial of a work-focused cognitive behavioral intervention

by Dalgaard VL, Aschbacher K, Andersen JH, Glasscock DJ, Willert MV, Carstensen O, Biering $\mathrm{K}$

In this randomized controlled trial (RCT) study, a work-focused cognitive behavioral therapy (CBT) treatment combined with an optional workplace intervention was associated with faster lasting return to work compared to a control group that received clinical assessment among patients on sick leave due to work-related stress. The intervention group returned to work four weeks faster, which could have substantial financial impact on both the employee and related societal costs.

Affiliation: Danish Ramazzini Centre, Dept. of Occupational Medicine, The Regional Hospital West Jutland - University Research Clinic, Gl. Landevej 61, 7400, Herning, Denmark. Vita.Ligaya.Dalgaard@vest.rm.dk

Refers to the following texts of the Journal: 2009;35(2):145-152 2011;37(3):186-195 2014;40(6):569-581 2016;42(4):273-279

The following article refers to this text: $2019 ; 45(2): 211-212$

Key terms: absenteeism; CBT; cognition; cognitive behavioral intervention; cognitive behavioral therapy; mental health problem; perceived stress; randomized controlled trial; RCT; rehabilitation; return to work; return-to-work; RTW; sick leave; sleep; stress; stress management; work stress; work-related stress

This article in PubMed: www.ncbi.nlm.nih.gov/pubmed/28650513

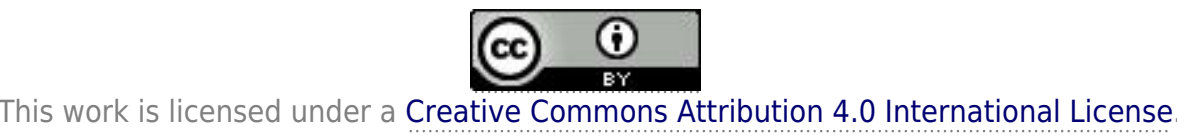




\title{
Return to work after work-related stress: a randomized controlled trial of a work-focused cognitive behavioral intervention
}

\author{
by Vita Ligaya Dalgaard, PhD, ${ }^{1}$ Kirstin Aschbacher, PhD, ${ }^{2}$ Johan Hviid Andersen, PhD, ${ }^{1}$ David John Glass- \\ cock, PhD, ${ }^{1}$ Morten Vejs Willert, PhD, ${ }^{3}$ Ole Carstensen, MD, ${ }^{1}$ Karin Biering, $P h D{ }^{1}$
}

\begin{abstract}
Dalgaard VL, Aschbacher K, Andersen JH, Glasscock DJ, Willert MV, Carstensen 0, Biering K. Return to work after work-related stress: a randomized controlled trial of a work-focused cognitive behavioral intervention. Scand J Work Environ Health. 2017;43(5):436-446. doi:10.5271/sjweh.3655
\end{abstract}

Objectives This study aimed to evaluate the effect of a stress management intervention (SMI) on lasting return to work (RTW) among patients with work-related stress complaints.

Methods Sickness benefit departments from three local municipalities referred patients on sick leave with work-related adjustment disorders or mild depression to the Department of Occupational Medicine, Regional Hospital West Jutland. A $2 \times$ randomization procedure allocated patients into one of three groups: intervention $(\mathrm{N}=58)$, control A (which received a clinical assessment; $\mathrm{N}=56$ ), or control $\mathrm{B}$ (no assessment; $\mathrm{N}=49$ ). Treatment comprised individual work-focused cognitive behavioral therapy (CBT) with an optional workplace intervention. The outcome was time until lasting RTW (16 and 44 weeks follow-up) using register data.

Results Median number of weeks until lasting RTW was 15, 19, and 32 for the intervention group, control A, and control B respectively. However, for group B, clinical assessment was not part of the inclusion process, which may have introduced selection bias. In the fully-adjusted Cox regression model, the intervention group exhibited significantly faster lasting RTW at 44 weeks; hazard ratio (HR) 1.57 [95\% confidence interval (95\% CI) 1.01-2.44] relative to control group A, with a non-significant trend evident at 16 weeks; HR 1.70 ( $95 \%$ CI 0.94 3.10), when controlling for age, gender, occupation, sick leave during previous year, full or partial sick leave, and diagnosis. Unadjusted analyses remained directionally consistent but were reduced to marginal significance.

Conclusions There was a tendency towards faster lasting RTW in the intervention group compared to control $\mathrm{A}$, which received clinical assessment, in all analyses. The intervention group returned to work about 4 weeks earlier than control A, which could translate into substantial financial gains.

Key terms absenteeism; cognition; CBT; cognitive behavioral therapy; mental health problem; perceived stress; RCT; rehabilitation; RTW; sick leave; sleep; stress management; work stress.

Work-related stress is a major health concern in the $21^{\text {st }}$ century (1), a common cause of sick leave $(2,3)$ and a substantial source of economic burden (4). Sickness absence may act as a global measure of workers' health that predicts lost productivity and disability pensions (5). Mental health problems are linked to about $45 \%$ of all sick leave cases in Denmark (6) at an estimated yearly cost of about $€ 7.5$ billion (4). Almost $30 \%$ of all sick leave days in Denmark have been estimated to be related to the psychosocial work environment (7), and some evidence suggests that this pattern is also applicable to other European countries (8).

The term "stress" refers to the experience of external demands exceeding personal coping resources, according to the transactional perspective of Lazarus \& Folkman (9). Stress may illicit psychological and physiological responses, which, over time, can lead to a variety of stress complaints and impaired functioning (10-12). Work-related stress may merit a unique treatment approach, which not only addresses thoughts and behaviors related to an individual's stress experience, but also the specific conditions at work.

1 Danish Ramazzini Centre, Dept. of Occupational Medicine, The Regional Hospital West Jutland - University Research Clinic, Herning, Denmark.

2 Division of Cardiology, School of Medicine, University of California, San Francisco, United States.

3 Danish Ramazzini Centre, Dept. of Occupational Medicine, Aarhus University Hospital, Aarhus, Denmark.

Correspondence to: Vita Ligaya Dalgaard, Danish Ramazzini Centre, Dept. of Occupational Medicine, The Regional Hospital West Jutland - University Research Clinic, Gl. Landevej 61, 7400, Herning, Denmark. [E-mail: Vita.Ligaya.Dalgaard@vest.rm.dk] 
During recent years, Danish departments of occupational medicine have seen a large increase in patients with work-related stress complaints. Many of these patients receive a diagnosis of adjustment disorder or reactions to severe stress [International Classification of Disease (ICD-10)] (13), and the majority are on part- or full-time sick leave. Typical stress complaints include cognitive difficulties, sleep problems, fatigue, tensions, muscular aches and feelings of sadness and anxiousness (14-17). Patients often report difficulties coping with work-related demands like high workload, role ambiguity, or social problems in the workplace.

In meta-analyses, cognitive behavioral therapy (CBT) is more effective at reducing psychological complaints among stressed workers than other stress management interventions (SMI) $(3,18,19)$. However, existing literature suffers from problems with sampling biases, lack of hard outcomes, and uncertain generalizability to the target population. For example, the majority of existing studies are based on volunteers from the workplace or stressed workers not on sick leave, and results may therefore not generalize to the broader population. Typically, only psychological measures are used as outcomes $(3,18,19)$. Less is known about effective treatments to facilitate return to work (RTW) among patients with clinically significant complaints $(3,18$, 19). Only a limited number of randomized controlled trials (RCT) have tested the effect of CBT based interventions for stressed workers on sick leave (10, 20-23).

The strongest effects on RTW have been shown by interventions that combine individual CBT with strategies that specifically target the work environment. For example, studies have combined CBT with a graded approach to RTW and work assignments $(20,21)$ or with workplace dialogue and mindfulness (23). Group-based CBT reduced self-reported absenteeism in one waitlist controlled study (22); however, other studies have not accelerated RTW by CBT using individual $(10,24)$ or group formats (10). A Cochrane meta-analysis reported that CBT was not superior to no treatment in reducing length of sick leave among workers with adjustment disorder. However, only nine studies were identified, with even fewer contributing to each meta-analysis (2). Differing results are likely influenced by inconsistencies in selection procedures, small sample sizes, and the lack of thorough diagnostic assessments at baseline to verify whether symptoms may in fact be understood as workrelated. In summary, it remains unclear if CBT-based SMI's speed up RTW in clinical samples with workrelated stress. However, studies that address the workplace in combination with CBT may hold more promise. We tested the efficacy of work-focused CBT combined with an optional workplace intervention for patients on sick leave due to work-related stress complaints, using a three-armed randomized controlled design. Stress arises as a function of an individual's personality and coping resources as well as the work environment. Thus, workfocused CBT targeted negative thoughts and maladaptive behaviors related to the individual's appraisal of work situations. Participants were offered an optional adjunctive intervention of having a psychologist participate in a workplace meeting. We employed two control groups as a more rigorous experimental design allowing us to contrast CBT treatment with a minimal, "care as usual", clinical assessment (control group A) versus a control group $\mathrm{B}$, which received neither the intervention nor the clinical assessment. We hypothesized that the intervention group would exhibit faster lasting RTW than either of the two control groups.

\section{Methods}

\section{Procedure and study design}

The study was designed as an RCT (trial number: ISRCTN91404229) with three arms. The 163 participants were randomly assigned into either the intervention group $(\mathrm{N}=58)$, control group $\mathrm{A}(\mathrm{N}=56)$ receiving clinical assessment but no treatment, or control group $\mathrm{B}(\mathrm{N}=49)$ receiving no offers at the department. A flowchart of recruitment, allocation, and data collection is depicted in figure 1. Uneven group sizes are explained below in procedures.

\section{Study population and recruitment procedures}

Patients were recruited to the Department of Occupational Medicine, Regional Hospital West Jutland through sickness benefit departments from three local municipalities. All patients were on sick leave (full or part time) due to work-related stress complaints.

\section{Screening questionnaire}

Potential participants $(\mathrm{N}=1182)$ were referred to the Department, and 52 individuals were excluded based on written referrals (see figure 1 for reasons). The remainder received a screening questionnaire and information about the trial. The questionnaire contained questions about employment, health, and whether stress symptoms were perceived as work-related. Participants who returned the questionnaire and met all inclusion criteria were invited to participate and received a letter of informed consent and a baseline questionnaire to be completed before allocation. Subsequently, participants were randomly assigned either to clinical assessment (required prior to assignment to intervention or control group A) or control group B (which received no clinical assessment) (see 


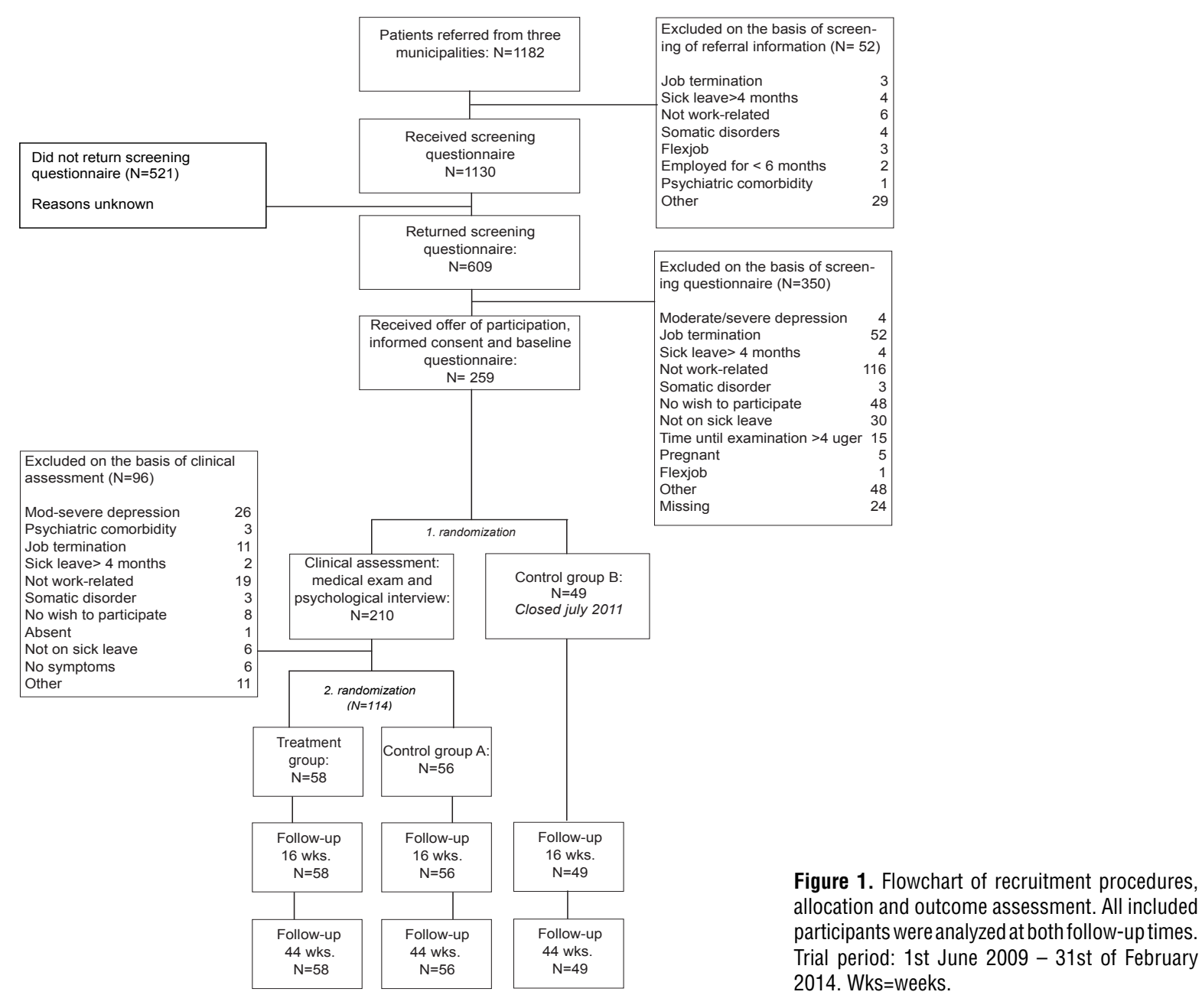

figure 1). During the inclusion period, we discovered that more participants than expected were excluded after clinical assessment for various reasons, eg, their condition was not sufficiently work-related (see figure 1 for more details). Therefore, we elected to stop randomization to control group B in July 2011 when it contained 49 participants. This meant that all new potential participants were invited to the clinical assessment on the basis of the screening questionnaire. Once inclusion and exclusion criteria had been assessed, participants were randomly assigned to either the intervention or control group A. Nonetheless, we report the results in accordance with the original study design, while acknowledging potential limitations related to control group B.

\section{Randomization}

Two randomization procedures were used as illustrated in the flowchart of the study (see figure 1). The study secretary conducted both. First, after returning the baseline questionnaire, each potential participant was given a number between 1-99999 generated by the True Random Number Generator (www.random.org) and randomized to either the clinical assessment or control group B (participants randomized to the clinical assessment: numbers 0-66666 and control group B: numbers 66667-99999). Second, participants included on the basis of the clinical assessment were randomized to either the intervention or control group A. These participants received a number from a list of 1000 randomly generated numbers between 0-100.000. Group assignment was based on the sum of the digits of this number (the intervention: unequal numbers and control group A: equal numbers)

\section{Inclusion and exclusion criteria}

Patients who received clinical examination were included if they fulfilled the following criteria: (i) a diagnosis of adjustment disorder or reactions to severe stress [ICD 10 code: F43.2-F43.9, but not PTSD (F43.1)] or mild depressive episode (F32.0) (13); (ii) on sick leave 
due to the above; (iii) the condition was evaluated by the psychologist as primarily work-related; (iv) patients planned on returning to their workplace. Exclusion criteria were: (i) comorbidity of another psychiatric illness (eg, moderate to severe depression), (ii) comorbidity of a recently diagnosed chronic somatic disease, (iii) pregnancy, (iv) substance abuse, (v) sick leave for $>4$ months prior to baseline, (vi) any degree of disability pension, (vii) employment $<6$ months; or (viii) job termination prior to baseline.

These exclusion criteria were used during all steps through the flow of the study (see figure 1) when the relevant information was available from the first referral through the screening questionnaire to the clinical assessment interview. Of course, some information such as diagnosis or a psychologist's evaluation of work-relatedness, was not available prior to clinical examination.

\section{The clinical examination}

An experienced occupational physician conducted a medical examination to rule out any somatic causes of symptoms (eg, diabetes, heart disease, thyroid disease). The medical examination followed a protocol, which covered education, social relations, health dispositions, psychiatric disease, alcohol, and medications.

Afterwards, a trained psychologist conducted a semistructured interview covering employment history; present work situation, including psychosocial work stressors; sick leave status; and symptom development and current symptoms. Non-work stressors were also addressed. The interview determined the diagnosis and assessed the likelihood that work-related stress played a primary role in symptom development. Patients were not excluded if stressors related to non-work conditions (like relationship or family problems) were present so long as they were of a secondary nature.

\section{Intervention}

The intervention consisted of six, one-hour sessions with individual work-focused CBT conducted by a psychologist over 16 weeks. The treatment protocol (available from the authors) was developed together with specialists in CBT specifically for patients with work-related stress complaints. The intervention aimed at reducing symptoms, strengthening coping ability, and facilitating RTW. This involved: (i) identifying work-related stressors, (ii) modifying cognitive and behavioral coping strategies, (iii) providing psycho-education about workrelated stress, (iv) assigning homework, and (v) assisting participants in planning RTW. Treatment was carried out according to protocol, but the psychologist had some flexibility in choosing among the listed techniques and homework assignments according to the patient's clinical diagnosis and individual needs. The psychologists took part in a short training program and received external supervision to ensure adherence to the protocol.

With regard to work resumption, patients were advised and assisted in planning gradual resumption. Work hours and task complexity was gradually increased to match the recovery stage of the patient. The intervention also offered a minor workplace intervention, comprised of one or two meetings at the workplace with the patient, the psychologist, a supervisor and/or a human resource representative. The aim was to address stressors at work and involve the workplace. The meetings addressed present stressors such as high workload or role ambiguity and provided suggestions for adjustment. The psychologist acted as an advisor to the workplace but had no decision authority. Only six participants $(10 \%)$ accepted the offer of psychologist participation in a workplace meeting. However, for those who preferred to have these discussions without the psychologist present, the psychologist provided support to the patient in preparing for the meeting. Meetings could take place at any time during the 16-week treatment period.

\section{Outcomes}

Lasting return to work: Register data. Time until lasting RTW was measured with data from the Danish national register on "social transfer payments" (the DREAM database). These payments include reimbursements to employers from the Danish government and other payments related to sick leave. DREAM contains information on all social transfer payments ( $>100$ types) to Danish citizens since 1991. The DREAM database contains a code for each transfer payment lasting $\geq 1$ day. Only one type of transfer payment code can be registered weekly (25); however, sickness absence coverage is always given the highest priority in the database. Hence, there is no missing data on sick leave payments so long as employers claim reimbursement. DREAM differentiates between major types of registration such as "sick leave" or "unemployment", although the database does not differentiate between full- or part-time sick leave (22).

To understand the DREAM database, it is useful to know some basic information about the Danish social security system. Danish employees normally receive full salary from their employers during sickness absence. However, employers become eligible to apply for partial reimbursement from the Danish state: (i) once a sick employee has been absent for $>2$ weeks (at time of the study, now longer), and (ii) so long as the employee in question has been employed for $\geq 8$ consecutive weeks prior to becoming sick (25).

If an employee is on sick leave but is fired from the 
workplace during his/her absence, instead of salary, the employee will receive general sickness absence compensation directly from the municipality. As long as a person receives any sort of sickness compensation, he/ she will only be registered in DREAM with a sickness payment code even if he/she has become unemployed (regardless of the cause). Thus, the outcome of "time until lasting RTW" is unaffected by unemployment as long as the person still receives sickness compensation. An unemployed (former employee) who stops receiving sickness benefits will subsequently transfer to a code for unemployment in DREAM up until eventual employment or other equivalent activities such as education. It is not possible to detect the reasons behind unemployment (ie, if a person was fired or resigned) in DREAM.

The coding of the DREAM registrations used for analyzing lasting RTW in this study was carried out in accordance with a previous Danish stress management study (22). Lasting RTW was defined as four consecutive weeks with no registration of sick leave payments or equivalent such as registrations related to educational grants. A registration in DREAM of sick leave, unemployment benefits, or certain types of rehabilitation benefits not involving job security were used as a negative outcome (ie, as equivalent to partial or full sick leave) in the analysis of lasting RTW. This means that those who transferred directly from sick leave to unemployment during the follow-up period were analyzed as not having returned to work up until eventual employment.

DREAM data were obtained for each participant from one year prior to baseline to 44 weeks after (ie, in accordance with a follow-up period of 10 months for the questionnaires used in the study).

Time until lasting RTW was a secondary outcome measure in the original trial. There was no intervention effect on primary outcomes perceived stress and general mental health as reported by Dalgaard et al [Work-focused cognitive behavioral intervention for psychological complaints in patients on sick leave due to work-related stress: Results from a randomized controlled trial (manuscript under review)].

Other data. Information on self-reported sick leave status (full or partial), length of sick leave, basic and higher education, occupation, and use of medication (table 1) were collected via the baseline questionnaire. Age and gender were generated from the participants' civil registration number (26), which was also connected to the DREAM register. Information on numbers of weeks on sick leave during the previous year was retrieved from the DREAM database. In addition, self-reported information on work-status was available from follow-up questionnaires administered at 4 and 10 months after baseline, equivalent to the follow-up times in registers of 16 and 44 weeks, respectively. The follow-up question-
Table 1. Demographic and baseline characteristics for all groups. [IT=information technology; SD=standard deviation.] a, b, c

\begin{tabular}{|c|c|c|c|c|c|c|}
\hline \multirow[t]{2}{*}{ Characteristics } & \multicolumn{2}{|c|}{ Intervention } & \multicolumn{2}{|c|}{ Control group A } & \multicolumn{2}{|c|}{ Control group B } \\
\hline & $\mathrm{N}$ & $\%$ & $\mathrm{~N}$ & $\%$ & $\mathrm{~N}$ & $\%$ \\
\hline \multicolumn{7}{|l|}{ Gender } \\
\hline Female & 43 & 74.1 & 40 & 71.4 & 37 & 75.5 \\
\hline Male & 15 & 25.9 & 16 & 28.6 & 12 & 24.5 \\
\hline \multicolumn{7}{|l|}{$\begin{array}{l}\text { Self-reported sick } \\
\text { leave at baseline }\end{array}$} \\
\hline Full & 33 & 56.9 & 35 & 62.5 & 25 & 51.0 \\
\hline Partial & 25 & 43.1 & 21 & 37.5 & 16 & 32.7 \\
\hline Missing values & & & & & 8 & 16.3 \\
\hline \multicolumn{7}{|l|}{ Basic education } \\
\hline $9^{\text {th }}$ grade or less & 8 & 13.8 & 5 & 8.9 & 3 & 6.1 \\
\hline 10-12 years & 49 & 84.5 & 50 & 89.3 & 39 & 79.6 \\
\hline Missing values & 1 & 1.7 & 1 & 1.8 & 7 & 14.3 \\
\hline \multicolumn{7}{|c|}{ Higher education (years) } \\
\hline Short $(<3)$ & 21 & 36.2 & 27 & 48.2 & 26 & 53.1 \\
\hline Medium (3-4) & 28 & 48.3 & 24 & 42.9 & 15 & 30.6 \\
\hline Long (>4) & 9 & 15.5 & 5 & 8.9 & 1 & 2.0 \\
\hline Missing values & & & & & 7 & 14.3 \\
\hline \multicolumn{7}{|l|}{ Occupation by field } \\
\hline Health & 5 & 8.6 & 5 & 8.9 & 6 & 12.2 \\
\hline Teaching & 7 & 12.1 & 8 & 14.3 & 3 & 6.1 \\
\hline Administration & 5 & 8.6 & 4 & 7.1 & 10 & 20.4 \\
\hline $\begin{array}{l}\text { Daycare/social } \\
\text { worker }\end{array}$ & 8 & 13.8 & 9 & 16.1 & 3 & 6.1 \\
\hline Leader & 11 & 19.0 & 8 & 14.3 & 6 & 12.2 \\
\hline Trade/banking/IT & 2 & 3.5 & 8 & 14.3 & 4 & 8.2 \\
\hline Other & 20 & 34.5 & 14 & 25.0 & 9 & 18.4 \\
\hline Missing values & & & & & 8 & 16.3 \\
\hline \multicolumn{7}{|l|}{ Diagnosis (ICD-10) } \\
\hline Mild depression & 16 & 27.6 & 11 & 19.6 & & \\
\hline $\begin{array}{l}\text { Adjustment } \\
\text { disorder/reactions } \\
\text { to stress }\end{array}$ & 42 & 72.4 & 45 & 80.4 & & \\
\hline Taking medication & 27 & 44.8 & 25 & 44.6 & 25 & 51.0 \\
\hline \multicolumn{7}{|l|}{$\begin{array}{l}\text { Medication } \\
\text { (by indication) }\end{array}$} \\
\hline Depression & 14 & 24.1 & 11 & 19.6 & 13 & 26.5 \\
\hline Anxiety & 4 & 6.9 & 5 & 8.9 & 1 & 2.0 \\
\hline Sleeping problems & 6 & 10.3 & 5 & 8.9 & 8 & 16.3 \\
\hline Other & 14 & 24.1 & 13 & 23.2 & 13 & 26.5 \\
\hline
\end{tabular}

a Mean age: intervention group = 45 (range 28-60); control group $A=44$ (range 29-63); control group $B=46$ (range 26-62) years.

b Mean days of self-reported sick leave at baseline: intervention group $=$ 77.6 ( $\mathrm{SD}=17,95 \% \mathrm{Cl} 73.0-82.2)$; control group $\mathrm{A}=74.9(\mathrm{SD}=16,95 \%$ Cl 70.6-79.3); control group B $=74.1$ (SD=31, 95\% Cl 63.9-84.4).

${ }^{c}$ Mean numbers of self-reported years in current job position: intervention group $=9$ (range $1-28) ;$ control group $A=9$ (range 1-42); and control group $B=9$ (range $0.5-31$ ) years.

naires also contained information on use of professional help in the control groups. Information on diagnoses for the intervention and control group A was registered by the psychologist at the time of clinical assessment.

\section{Statistical analysis}

Statistical analyses were conducted in STATA (Stata Corp LP, College Station, TX, USA) software package 13.1. Baseline characteristics were compared using descriptive statistics and all data was analyzed as intention to treat. 
Time until lasting RTW was measured from inclusion into the study. Group differences in time until lasting RTW were illustrated by drawing a Kaplan-Meier plot of the cumulative probability of being on full or part time sick leave and carrying out two Cox regression analyses with 16 and 44 weeks follow-up, respectively. Crude and adjusted analyses were conducted. Based on existing literature, the following confounders were evaluated as most relevant prior to conducting analyses: gender, age, occupation at baseline, weeks of sick leave during the previous year, self-reported sick leave status at baseline (full or partial), and diagnosis (not applicable to group B). The occupation variable was divided into the following: health personnel, daycare and social workers in one group and all others in the other group (ie, caretakers versus none caretakers). The adjusted models were divided into the following: age and gender (model 1); age, gender, and weeks in DREAM the previous year before inclusion, occupation at baseline (caretaking and health personnel versus other occupations), self-reported sick leave status at baseline (full or partly) (model 2); covariates given in model 2 plus diagnoses - adjustment disorders and reactions to severe stress versus mild depression (model 3). Control group B could not be included in model 3 due to lack of diagnostic data.

Model validation of the proportional hazards assumption was performed by conducting a log-log plot of the survival curves and the proportional hazards test with and without covariates.

Participants still on sick leave at each follow-up measurement were right censored in the Cox regression analyses. One participant transferred directly from sick leave to early retirement and was therefore censored at 25 weeks follow-up when retirement occurred.

\section{Results}

\section{Baseline characteristics}

Demographic and baseline characteristics for the intervention and two control groups are presented in table 1. Baseline values were compared with descriptive statistics.

At baseline, all participants were registered in DREAM as on sick leave, either part- or full time, with the exception of one person in control group B, who had resumed work only days after inclusion as shown by information from the questionnaire data. She was kept in the analyses in accordance with the intentionto-treat principle and with a time until lasting RTW of one week.

\section{Rates of lasting return to work}

The median and restricted mean values of time until lasting RTW are stated in table 2 . It can be seen that median time to lasting RTW in the intervention group was 4 weeks shorter than control group A (corresponding to a median ratio of 1.27) and 17 weeks shorter than control group B (corresponding to a median ratio of 2.13).

The cumulative probability of being partly/fully on sick leave or unemployed (ie, no longer on sick leave but without employment) after baseline is presented in a Kaplan-Meier plot (see figure 2) with 44 weeks followup for all three groups.

In table 3, the intention-to-treat analyses comparing all three groups and using control group A as the reference are presented. At 16 weeks follow-up, crude results favored the intervention group, although differences did not reach statistical significance. The hazard ratio (HR) and confidence intervals (CI) improved in favor of the

Table 2. Register-based median and mean numbers of weeks until lasting return to work (RTW) in the intervention, control group $\mathrm{A}$, and control group $\mathrm{B}$ at 44 weeks follow-up. [ $95 \% \mathrm{Cl}=95 \%$ confidence intervals.]

\begin{tabular}{|c|c|c|c|c|c|c|}
\hline & \multicolumn{2}{|c|}{ Intervention } & \multicolumn{2}{|c|}{ Control group A } & \multicolumn{2}{|c|}{ Control group B } \\
\hline & weeks & $95 \% \mathrm{Cl}$ & weeks & $95 \% \mathrm{Cl}$ & weeks & $95 \% \mathrm{Cl}$ \\
\hline Median a & 15 & & 1 & ) & 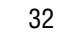 & \\
\hline Restricted mean ${ }^{b}$ & 20 & $17-24$ & 25 & $21-28$ & 29 & $5-$ \\
\hline
\end{tabular}

a The upper part of the $95 \% \mathrm{Cl}$ could not be reported in group B due to the high number of participants that had not returned to work at 44 weeks follow-up in this group.

b 11 participants in the intervention group, 11 participants in the control group and 18 participants in control group B were censored at 44 weeks since they had not returned to work and therefore mean length until lasting RTW is underestimated.

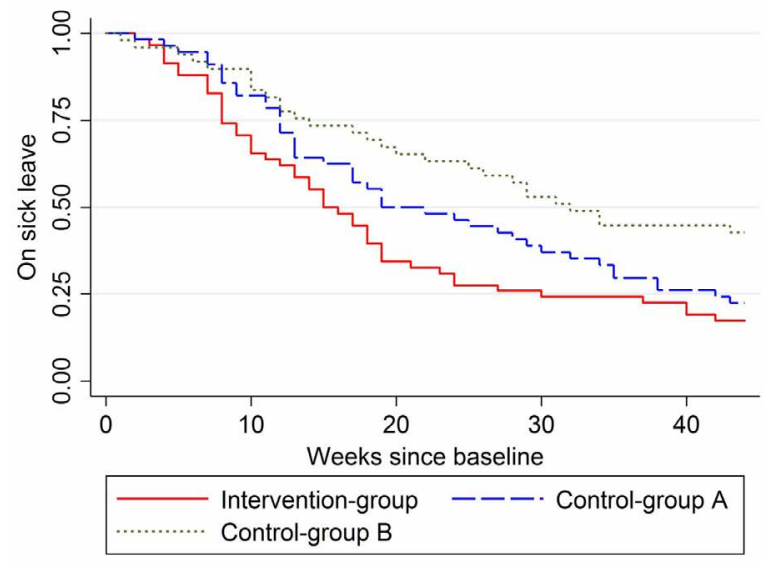

Figure 2. Kaplan-Meier plot of rates of lasting return to work represented by the cumulative probability of being on part or full time sick leave (or equivalent). Lasting return to work was defined as four consecutive weeks not registered with sick leave in DREAM. 
intervention group when adjusting for gender, age, number of weeks on sick leave the previous year, self-reported sick leave status (full or partial) at baseline, occupation as well as diagnosis (not applicable to group B).

The intention-to-treat analyses comparing all three groups at 44 weeks follow-up are presented in table 4 . The crude results were in favor of the intervention group when compared to control group A although they were not statistically significant. Adjusting for potential confounders increased estimates in favor of the intervention.

Many participants reported in questionnaires at 10 months follow-up that they had been fired at some point during the study period (11 in the intervention group, 10 in control group A, and 16 in control group B). Others reported resigning ( 3 people in the intervention group, 4 in control group A, and 8 in control group B). However, 6 in the intervention group, 10 in control group A and 7 in control group B reported that they had been hired in new positions. The exact time of initiation of unemployment was not available in the self-report measures. However, in the DREAM database, it was visible that unemployment only influenced the outcome of lasting RTW in a few cases partly because many participants, who reported that they had been fired remained sick and were thus coded as receiving sickness compensation in DREAM through to the end of the study.

Several sensitivity analyses were conducted to test the robustness of the results as presented in the following sections. We conducted one sensitivity analysis by removing three participants in the intervention group and control group A, who transferred directly from sick leave to unemployment in the DREAM database and remained unemployed. This did not significantly change the size or the direction of the estimates [HR $1.51(95 \% \mathrm{CI}$ 0.97-2.34) $\mathrm{P}=0.06$ ] at 44 weeks follow-up. Additionally, a sensitivity analysis removing the six participants who received a workplace intervention was carried out. This enhanced the estimates in the fully adjusted model at 44 weeks follow-up [HR 1.86 (95\% CI 1.18-2.92), $\mathrm{P}=0.007$ ].

The covariates were chosen based on existing literature, while complying with the conservative rule of thumb of $\geq 10$ events per covariate. The occupation variable, used in models 2 and 3 was dichotomized into two categories to minimize the number of covariates at 16 weeks follow-up. In the follow-up measurement at 44 weeks, there were more events, and therefore there was room for more covariates. So in addition to the result at 44 weeks in model 3 (see table 4), we also conducted a sensitivity analysis using the occupation variable with six categories, closely resembling the original categories as presented in table 1 . There were 91 events in the final adjusted model (not applicable to group B) including all participants in the intervention group and control group $\mathrm{A}$, and there were 10 covariates with the new occupation variable. This analysis suggested an even stronger intervention effect [HR $1.79(95 \%$ CI 1.13-2.82) $\mathrm{P}=0.01$ ].

Six psychologists participated in the study. A subanalysis revealed no significant differences with regard to lasting RTW rates between psychologists. Participants in the control groups were free to seek psychological assistance elsewhere and many chose to do so (36 in control group A, 28 in control group B). Only 4 participants in control group A and 2 in control group B reported that they had not received assistance from a

Table 3. Hazard ratios (HR) of analyses of time until lasting return to work at 16 weeks follow-up. [95\% $\mathrm{Cl}=95 \%$ confidence intervals.]

\begin{tabular}{|c|c|c|c|c|c|c|c|c|c|c|c|c|}
\hline \multirow[t]{2}{*}{ Group } & \multicolumn{3}{|c|}{ Crude model } & \multicolumn{3}{|c|}{ Adjusted model $1^{\mathrm{a}}$} & \multicolumn{3}{|c|}{ Adjusted model $2 \mathrm{~b}$} & \multicolumn{3}{|c|}{ Adjusted model $3^{\mathrm{c}}$} \\
\hline & $\mathrm{HR}$ & $95 \% \mathrm{Cl}$ & P-value & $\mathrm{HR}$ & $95 \% \mathrm{Cl}$ & P-value & $\mathrm{HR}$ & $95 \% \mathrm{Cl}$ & P-value & $\mathrm{HR}$ & $95 \% \mathrm{Cl}$ & $P$-value \\
\hline Intervention & 1.50 & $0.85-2.62$ & 0.16 & 1.56 & $0.89-2.75$ & 0.12 & 1.57 & $0.87-2.82$ & 0.13 & 1.70 & $0.94-3.10$ & 0.08 \\
\hline Control A & 1 & & & 1 & & & 1 & & & 1 & & \\
\hline Control B & 0.68 & $0.34-1.36$ & 0.28 & 0.70 & $0.35-1.42$ & 0.33 & 0.66 & $0.31-1.42$ & 0.29 & & & \\
\hline
\end{tabular}

adjusted for age and gender.

${ }^{\mathrm{b}}$ Model 1 plus weeks in DREAM the previous year before inclusion, occupation at baseline (caretaking and health personnel vs. other occupations) selfreported sick leave status at baseline (full or partly).

c Model 2 plus diagnosis. Control group B was omitted in model 3 due to lack of diagnosis and reducing number of events to 49.

Table 4. Hazard ratios of analyses of time until lasting return to work at 44 weeks follow-up. [ $95 \% \mathrm{Cl}=95 \%$ confidence intervals.]

\begin{tabular}{|c|c|c|c|c|c|c|c|c|c|c|c|c|}
\hline \multirow[t]{2}{*}{ Group } & \multicolumn{3}{|c|}{ Crude model } & \multicolumn{3}{|c|}{ Adjusted model $1^{\mathrm{a}}$} & \multicolumn{3}{|c|}{ Adjusted model $2{ }^{\mathrm{b}}$} & \multicolumn{3}{|c|}{ Adjusted model $3 \mathrm{c}$} \\
\hline & $\mathrm{HR}$ & $95 \% \mathrm{Cl}$ & P-value & $\mathrm{HR}$ & $95 \% \mathrm{Cl}$ & P-value & $\mathrm{HR}$ & $95 \% \mathrm{Cl}$ & P-value & $\mathrm{HR}$ & $95 \% \mathrm{Cl}$ & $\mathrm{P}$-value \\
\hline Intervention & 1.33 & $0.88-2.01$ & 0.17 & 1.36 & $0.89-2.05$ & 0.15 & 1.44 & $0.94-2.21$ & 0.09 & 1.57 & $1.01-2.44$ & 0.04 \\
\hline Control A & 1 & & & 1 & & & 1 & & & 1 & & \\
\hline Control B & 0.61 & $0.38-1.00$ & 0.05 & 0.62 & $0.39-1.01$ & 0.05 & 0.60 & $0.36-1.00$ & 0.05 & & & \\
\hline
\end{tabular}

${ }^{a}$ Adjusted for age and gender

${ }^{\mathrm{b}}$ Model 1 plus weeks in DREAM the previous year before inclusion, occupation at baseline (caretaking and health personnel vs. other occupations) selfreported sick leave status at baseline (full or partly).

c Model 2 plus diagnosis. Control group B was omitted in model 3 due to lack of diagnosis. Number of events: 91. 
psychologist, doctor, coach or psychiatrist during the first 16 weeks of the study.

\section{Discussion}

Patients in the intervention group resumed work around 4 weeks earlier than control group A, which received a clinical assessment. The difference was statistically significant in the fully adjusted model at 44 weeks follow-up and showed a marginal tendency in favor of the intervention in all additional models. While some limitations exist in the current study, the results strongly suggest the possibility that interventions for work-related stress conditions could facilitate earlier RTW and save costs related to sickness benefits and individual financial loss.

\section{Comparison to previous studies}

Our results broadly resemble those of a few other SMI studies conducted with similar samples. In general, the majority of participants return to work within 10 months, both in this study and in prior studies $(10,22$, 24 ), suggesting that stress conditions naturally resolve with a certain timeframe. However, $23 \%$ of all participants and $18 \%$ of the participants in the intervention group and group A were among those fired at some point during the study period. This indicates that sick leave due to work-related stress may involve a high risk of losing one's job with negative financial and social implications for the individual. As found in other studies (11, 24 ), about $20-25 \%$ in the intervention and control group A were still on sick leave towards the end of the study and many had been fired. Thus, this remaining group may experience increased risk of elimination from the workforce and could be in need of more extensive help in the process of returning to work. Additionally, becoming unemployed while on sick leave could potentially contribute to worsening of the condition of the patient for example in terms of worries or anxiety about one's future work situation. However, the sensitivity analysis, where we removed individuals in the intervention and control group A, who transferred directly from sick leave to unemployment in DREAM, indicates that the overall results were not influenced by the occurrence of unemployment (regardless of the cause). Yet, it cannot be ruled out that unemployment may have reduced the effect of our intervention and potentially made it difficult for some participants to benefit from the work focus of the intervention.

In the cluster-randomized study by van der Klink et al (20), the combination of cognitive behavioral and gradual activity programs was superior to care as usual (ie, meeting with a resident occupational physician) in reducing absenteeism and increasing initial RTW. The studies by both Blonk et al (21) and van der Klink et al (20) differed from ours on one key factor: the degree to which the intervention was able to directly address the workplace. More specifically, they both implemented a relatively short CBT program with a direct workplace approach addressing numerous work factors and graded RTW. We incorporated most of these components into the current intervention; however, the majority of participants in our study declined the optional workplace intervention. This meant that the psychologist did not have the same ability to participate in the workplace dialogue. The lack of direct workplace contact may therefore have limited the impact of our intervention. In a comparative study, Lagerveld et al (27) compared work-focused CBT to regular CBT for employees on sick leave due to common mental health disorders. In the work-focused intervention, CBT techniques were applied to the work context in combination with graded activity principles. Similar to the current study, the group that received work-focused CBT returned to full work resumption 65 days prior to the comparison group. The intervention used by Lagerveld et al (27) is similar to our intervention although the authors employed more sessions and included a broader sample of milder mental health conditions.

The current intervention was previously tested in a study employing a two-armed RCT design with a different selection procedure (ie, patients were referred directly from their general practitioner (GP) as opposed to municipalities in the current study). In that study, Glasscock et al [Recovery from workrelated stress: A randomized controlled trial of a stress management intervention in a clinical sample (manuscript in preparation)] found no difference in rates of lasting RTW between the intervention group and a no-treatment control group (similar to the present control group A). It is notable that the mean number of self-reported days on sick leave at inclusion was almost twice as high in the current study than in the previous study. Therefore, on average, the current sample experienced a longer recovery period prior to inclusion in the study. Gradual RTW was an integral part of this study's intervention. Some have advocated gradual RTW across a broad spectrum of conditions (28). However, when sick leave is related to mental health issues (29), ours and other findings indicate that there may be a "window of opportunity" for optimal intervention after the first 60 days. One study found that gradual RTW was associated with a positive outcome (ie, recovery of work ability), only when initiated among mental health patients who had been on sick leave for $\geq 60$ days (29). On the one hand, early treatment is often recommended for 
patients on sick leave due to mental health conditions (4), and long-term sickness absence is associated with increased risk of extinction from the work force (8). On the other hand, this study's results suggest that some patients may require at least a couple months of recovery before they can benefit from gradual RTW. Alternatively, patients who do not return to work on their own after a relatively short sick leave period may have more severe adjustment disorders and may therefore show more benefit from a tailored intervention.

\section{Generalizability}

Our study sample is demographically comparable to those of other Danish SMI studies (22-24, 30). It was comprised of mostly women in their mid-forties employed in the public sector. This could potentially reflect that many public institutions have experienced financial cuts during recent years that have disproportionately affected caretaking roles like nurses and daycare workers, occupations where a majority of workers are female. Thus, the findings may be less applicable to men or adults of other age groups. In addition, Danish sick-leave regulations and administrative policies may differ from those of other countries, thereby limiting generalizability.

\section{Strengths and limitations}

The RCT design and the use of lasting RTW as an outcome are major strengths of the current study. The register data was available for all participants and were not influenced by reporting bias or loss to follow-up. Lasting RTW was defined as four consecutive weeks with no transfer payment (such as health-related benefits) in DREAM, based on two prior stress management studies $(22,30)$ and on a previous study from Biering et al (31). If one used only the first week with no transfer payment indicating sick leave, then temporary administrative breaks, such as holidays or payment changes, could easily be misinterpreted as RTW. Studies often examine RTW without clearly specifying what is meant by the term full or lasting RTW $(10,20,23)$. Biering et al (31) advocated the use of registers like DREAM as an opportunity to use a measure reflecting sustainable work resumption (ie, in this case four consecutive weeks of work resumption).

Our study also has limitations. All patients underwent thorough assessment at baseline to determine suitability and ensure the possible work-relatedness of their stress condition. A number of patients were excluded during this process, suggesting that a similar selection would have happened if control group B had also been thoroughly assessed. The difference in outcomes between group B and the two other groups were thus most likely due to selection biases. Since group B was not clinically assessed at baseline, it is more likely to have included individuals with moderate-to-major depression or other more serious psychological conditions, as well as participants with non-work-related conditions. Hence, future intervention studies examining RTW should select all participants on the basis of a clinical assessment, regardless of condition. Often subjects are selected primarily on the basis of their own perceptions of being stressed by work. The assessment procedure used in the present study showed that although social workers from the referring municipalities and the patient may attribute his or her condition to workrelated stress, this may not be the case when examined by experts. Selection biases may also have occurred during the screening process (see figure 1), since many potential participants did not return the screening questionnaire. We do not know if these individuals did not have the energy to participate (ie, too stressed to get dressed), so it is possible that our sample may represent a subgroup with better coping resources.

Another limitation concerns the use of professional help (psychologist or general practitioner) in control group A (and B). The fact that many patients in the control groups sought treatment outside of the study context may reduce chances of finding treatment effects. The control groups might therefore best be understood as traditional treatment-as-usual groups. On the other hand, this enhances the study's external validity since most individuals on sick leave in Denmark are in contact with various health professionals. RCT within the area of work-related mental health are quite rare and involve ethical and practical challenges regarding control groups, and true no-treatment control groups are hard to establish since it is not ethically feasible to ask control groups not to seek help elsewhere.

The DREAM register covers entries on sick leave compensations. These entries depend on employers claiming the compensation refund and may therefore be prone to some level of imprecision, although Statistics Denmark reports that DREAM represents the $100 \%$ true levels of granted compensation for sickness absence, disability pensions and early retirement pension (www. dst.dk). DREAM only included sick leave spells of $>2$ weeks and is, therefore, not a sensitive measure of short-term absences. However, this is less relevant in the case of sick leave due to stress that is mostly of a longer duration. Unfortunately, DREAM does not distinguish between full- and part-time sick leave, which means that we could not identify the point in time where an employee returns partially to work with reduced working hours and tasks. This implies that some of those who had not returned fully at the end of the follow-up period may have returned to work on reduced hours. However, questionnaire data suggest that this was not 
the case, showing that many who were still on sick leave in DREAM at 44 weeks follow-up reported that they were still on fulltime sick leave with the majority also reporting that they had been fired from their jobs as previously discussed. Most likely the length of sick leave was an incentive to fire participants but other factors such as type of employment contract or position of the employee may also have played a role.

As in other Danish SMI addressing both the stressed individual and the workplace, only a small percentage $(10 \%)$ of the intervention group agreed to receive a direct workplace intervention. Contrary to the studies by Blonk et al (21) and van der Klink et al (20), Danish studies have only achieved workplace contacts of $19 \%$ (23) and $25 \%$ of the participants (15). Bringing a psychologist into the workplace may raise concerns about mental health stigma. However, the psychologists working in the study reported that patients became skilled in communicating with the workplace through the individual sessions and, therefore, preferred to do this on their own. Finally, as in other SMI studies, we cannot rule out that the relatively small sample size, which was mainly due to exclusion of participants during the clinical assessment, has influenced the results.

\section{Concluding remarks}

The combination of a work-focused CBT treatment with an optional workplace intervention was associated with faster lasting RTW compared to a control group that received a similar initial clinical assessment. The intervention group returned to work roughly four weeks faster than a control group offered a clinical assessment. Work-focused CBT treatments, which accelerate RTW may substantially reduce costs for employers and employees and reduce the burden on the medical system.

\section{Acknowledgements}

We would like to thank all the study participants, occupational physicians, psychologists, the project secretary and the data manager for their efforts in the current study. Furthermore, we would like to thank the participating municipalities of Herning, Ringkjøbing-Skjern, and Ikast-Brande.

\section{Funding}

The Danish Working Environment Research Fund supported this work (grant number 15-2009-03). The second author's contribution to the study was supported by NIH/NHLBI grant K23 HL112955 and the Institute for Integrative Health.

\section{Ethics}

The study was approved by the Danish Data Protection Agency. Since the study classifies as a survey, approval from the Research Ethics Committee was not required.

\section{References}

1. Houtman I, Jettinghoff K, Cedillo L. Protecting Workers' Health Series No. 6. Raising awareness of stress at work in developing countries. 2007;(ISBN 924159165 X).

2. Arends I, Bruinvels DJ, Rebergen DS, Nieuwenhuijsen K, Madan I, Neumeyer-Gromen A, et al. Interventions to facilitate return to work in adults with adjustment disorders. Cochrane Database Syst Rev. 2012 Dec 12;12:CD006389. https://doi. org/10.1002/14651858.cd006389.pub2.

3. van der Klink JJ, Blonk RW, Schene AH, van Dijk FJ. The benefits of interventions for work-related stress. Am J Public Health. 2001 Feb;91(2):270-6. https://doi.org/10.2105/ AJPH.91.2.270.

4. Borg V, Andersen Nexø M, Kolte IV, Friss Andersen M Hvidbog om mentalt helbred, sygefravær og tilbagevenden til arbejde [White paper on mental health, sickness absence and return to work]. København: Det Nationale Forskningscenter for Arbejdsmiljø; 2010.

5. Kivimaki M, Head J, Ferrie JE, Shipley MJ, Vahtera J, Marmot MG. Sickness absence as a global measure of health: evidence from mortality in the Whitehall II prospective cohort study. BMJ. 2003 Aug 16;327(7411):364. https://doi.org/10.1136/ bmj.327.7411.364.

6. Søefeldt MB, Hallin C. Mental health problems and sick leave. Copenhagen: Danish Mental Health Fund; 2011.

7. Nielsen ML, Rugulies R, Smith-Hansen L, Christensen KB, Kristensen TS. Psychosocial work environment and registered absence from work: estimating the etiologic fraction. Am J Ind Med. 2006 Mar;49(3):187-96. https://doi.org/10.1002/ ajim. 20252 .

8. Henderson M, Glozier N, Holland Elliott K. Long term sickness absence. BMJ. 2005 Apr 9;330(7495):802-3. https:// doi.org/10.1136/bmj.330.7495.802.

9. Lazarus RS, Folkman S. Stress, appraisal, and coping. New York (NY): Springer; 1984.

10. de Vente W, Kamphuis JH, Emmelkamp PM, Blonk RW. Individual and group cognitive-behavioral treatment for workrelated stress complaints and sickness absence: a randomized controlled trial. J Occup Health Psychol. 2008 Jul;13(3):214 31. https://doi.org/10.1037/1076-8998.13.3.214.

11. de Vente W, Kamphuis JH, Blonk RW, Emmelkamp PM. Recovery of Work-Related Stress: Complaint Reduction and Work-Resumption are Relatively Independent Processes. J Occup Rehabil. 2015 Sep;25(3):658-68. https://doi. org/10.1007/s10926-015-9573-6.

12. Mauss D, Li J, Schmidt B, Angerer P, Jarczok MN. Measuring 
allostatic load in the workforce: a systematic review. Ind Health. 2015;53(1):5-20. https://doi.org/10.2486/ indhealth.2014-0122.

13. The ICD-10 Classification of Mental and Behavioural Disorders. Clinical Descriptions and Diagnostic guidelines. Geneva: WHO; 1992.

14. Willert MV, Thulstrup AM, Hertz J. Changes in stress and coping from a randomized controlled trial of a three-month stress management intervention. Scand J Work Environ Health. 2009 Mar;35(2):145-52. https://doi.org/10.5271/sjweh.1313.

15. Dalgaard L, Eskildsen A, Carstensen O, Willert MV, Andersen JH, Glasscock DJ. Changes in self-reported sleep and cognitive failures: a randomized controlled trial of a stress management intervention. Scand J Work Environ Health. 2014 Nov;40(6):569-81. https://doi.org/10.5271/sjweh.3460.

16. Eskildsen A, Andersen LP, Pedersen AD, Vandborg SK, Andersen JH. Work-related stress is associated with impaired neuropsychological test performance: a clinical cross-sectional study. Stress. 2015;18(2):198-207. https://doi.org/10.3109/10 253890.2015 .1004629 .

17. Eskildsen A, Andersen LP, Pedersen AD, Andersen JH. Cognitive impairments in former patients with work-related stress complaints - one year later. Stress. 2016 Aug 31;(6):1-8. https://doi.org/10.1080/10253890.2016.1222370.

18. Richardson KM, Rothstein HR. Effects of occupational stress management intervention programs: a meta-analysis. J Occup Health Psychol. 2008 Jan;13(1):69-93. https://doi. org/10.1037/1076-8998.13.1.69.

19. Bhui KS, Dinos S, Stansfeld SA, White PD. A synthesis of the evidence for managing stress at work: a review of the reviews reporting on anxiety, depression, and absenteeism. J Environ Public Health. 2012;2012:515874. https://doi. org/10.1155/2012/515874.

20. van der Klink JJ, Blonk RW, Schene AH, van Dijk FJ. Reducing long term sickness absence by an activating intervention in adjustment disorders: a cluster randomised controlled design. Occup Environ Med. 2003 Jun;60(6):429-37. https://doi. org/10.1136/oem.60.6.429.

21. Blonk RWB, Brenninkmeijer V, Lagerfeld SE. Return to work: A comparison of two cognitive behavioural interventions in cases of work-related psychological complaints among the self-employed. Work Stress 2006;20:2:129-44. https://doi. org/10.1080/02678370600856615.
22. Willert MV, Thulstrup AM, Bonde JP. Effects of a stress management intervention on absenteeism and return to workresults from a randomized wait-list controlled trial. Scand J Work Environ Health. 2011 May;37(3):186-95. https://doi. org/10.5271/sjweh.3130.

23. Netterstrom B, Friebel L, Ladegaard Y. Effects of a multidisciplinary stress treatment programme on patient return to work rate and symptom reduction: results from a randomised, wait-list controlled trial. Psychother Psychosom. 2013;82(3):177-86. https://doi.org/10.1159/000346369.

24. Lander F, Friche C, Tornemand H, Andersen JH, Kirkeskov L. Can we enhance the ability to return to work among workers with stress-related disorders? BMC Public Health. 2009 Oct 5;9:372. https://doi.org/10.1186/1471-2458-9-372.

25. Lund T LM. Sickness absence in Denmark - research, results, and reflections. SJWEH Suppl. 2009;(7):5-14.

26. Schmidt M, Pedersen L, Sørensen HT. The Danish Civil Registration system as a tool in epidemiology. Eur J Epidemiol. 2014;29(8):541-9. https://doi.org/10.1007/ s10654-014-9930-3.

27. Lagerveld SE, Blonk RW, Brennink V, de Meij LW, Schaufeli WB. Work-focused Treatment of Common Mental Disorders and Return to Work: A Comparative Outcome Study. J Occup Health Psychol. 2012. Apr; 17(2):220-34.

28. Bethge M. Effects of graded return-to-work: a propensityscore-matched analysis. Scand J Work Environ Health. 2016 Jul 1;42(4):273-9. https://doi.org/10.5271/sjweh.3562.

29. Andrén D. Does part-time sick leave help individuals with mental disorders recover lost work capacity? J Occup Rehabil. 2014(24):344-60. https://doi.org/10.1007/s10926-013-94674.

30. Willert MV, Wieclaw J, Thulstrup AM. Rehabilitation of individuals on long-term sick leave due to sustained stress-related symptoms: a comparative follow-up study. Scand J Public Health. 2014 Dec;42(8):719-27. https://doi. org/10.1177/1403494814551859.

31. Biering K, Hjollund NH, Lund T. Methods in measuring return to work: a comparison of measures of return to work following treatment of coronary heart disease. J Occup Rehabil. 2013 Sep;23(3):400-5. https://doi.org/10.1007/s10926-0129405-x.

Received for publication: 23 November 2016 